\title{
Avaliação das posições e situações anatômicas do apêndice em pacientes pediátricos com apendicite aguda
}

\author{
Evaluation of the anatomic positions and situations of the appendix in \\ pediatric patients with acute appendicitis
}

\author{
Arthur Almeida Aguiar ${ }^{1}$, Maria Gabriela Dourado de Melo Gusmão², Amanda Leão Lins e \\ Mello², Bruna Almeida Andrade Velloso², Carolina Vanderley Menezes D’Almeida², \\ Raquel Nogueira Cordeiro ${ }^{2}$, Diego Laurentino Lima ${ }^{3}$
}

\begin{abstract}
Aguiar AA, Gusmão MGDM, Lins e Melo APL, Velloso BAA, D’Almeida CVA, Cordeiro RN, Lima DL. Avaliação das posições e situações anatômicas do apêndice em pacientes pediátricos com apendicite aguda / Evaluation of the anatomic positions and situations of the appendix in pediatric patients with acute appendicitis. Rev Med (São Paulo). 2019 jan.-fev.;98(1)1-7.
\end{abstract}

RESUMO: Objetivo: Avaliar as diferentes posições e situações anatômicas do apêndice em pacientes pediátricos com apendicite aguda. Método: Estudo observacional do tipo corte transversal, realizado em Agosto de 2015 a Julho de 2016, na Emergência Pediátrica do Hospital da Restauração, na cidade do Recife. A amostra foi composta por 56 pacientes na faixa etária de 7 a 13 anos diagnosticados com apendicite aguda. Os dados clínico-epidemiológicos dos participantes foram obtidos antes do procedimento cirúrgico. Durante a cirurgia, foram coletadas as características anatômicas do apêndice (posição, situação, comprimento e fase da apendicite). Resultados: As posições encontradas foram pélvica $(37,5 \%)$, retrocecal $(28,6 \%)$, pré-ileal (10,7\%), pós-ileal $(8,9 \%)$, subcecal $(8,9 \%)$ e paracecal $(5.4 \%)$. Quanto à situação, a mais vista foi descendente $(46,4 \%)$, seguida por ascendente $(28,6 \%)$, interna $(19,6 \%)$ e externa $(5,4 \%)$. As principais manifestações clínicas observadas foram dor em fossa ilíaca direita, vômitos e náuseas, independentemente da posição. Verificou-se que a fase inflamatória da apendicite foi a mais frequente em todas as posições, exceto na subcecal com $60 \%$ dos apêndices na fase perfurada. No entanto, não houve associação estatisticamente significante entre a posição subcecal e a fase da apendicite complicada $(\mathrm{p}=0,367)$. Conclusão: A posição pélvica e a situação descendente foram as mais frequentes na população de estudo. Não houve associação estatisticamente significante da posição do apêndice com a fase da apendicite e nem com o quadro clínico.

Descritores: Apendicite; Pediatria; Apêndice; Variação anatômica.

\begin{abstract}
Objective: to access the different anatomic positions and situations of the appendix in pediatric patients diagnosed with acute appendicitis. Method: It's an observational, cross-sectional study performed between august 2015 and July of 2016, at the Pediatric Emergency of the Restauration Hospital, in Recife, Brazil. The sample was composed of 56 patients from 7 to 13 years-old diagnosed with acute appendicitis. The clinic-epidemiologic data of the participants were collected before the surgical procedure. During the surgery, all anatomic characteristics of the appendices were gathered. (position, situation, length, period of the appendicitis). Results: The positions were: pelvic $(37.5 \%)$, retrocecal $(28.6 \%)$, pre-ileal $(10.7 \%)$, postileal $(8.9 \%)$, subcecal $(8.9 \%)$, and paracecal (5.4\%). Regarding situation, the most common was the descending (46.4\%), followed by the ascending (28.6\%), internal (19.6\%), and external (5.4\%). The main clinical manifestations observed were: pain in the lower right quadrant, vomits, and nausa, not related to the position of the appendix. The inflammatory period was the most frequent in all positions, except in the subcecal, with $60 \%$ being perforated. However, there was no statistically significant association between the subcecal position and the period of the complicated appendicitis $(\mathrm{p}=0.367)$. Conclusion: The pelvic position and the descending situation were the most frequent in the studied population. There was no statistically significant association between the position of the appendix and the period of the appendicitis, and neither with the clinical case.
\end{abstract}

Keywords: Appendicitis; Pediatrics; Appendix; Anatomical variation.

1 Médico cirurgião-pediátrico do Instituto de Medicina Integral Prof. Fernando Figueira - IMIP e Tutor de Anatomia da Faculdade Pernambucana de Saúde - FPS. Email: arthuraguiar@hotmail.com.

2. Acadêmica de Medicina da Faculdade Pernambucana de Saúde (FPS), Recife, Brasil.Email: bi gusmao@hotmail.com, amandalins@hotmail. com, bru.velloso@hotmail.com, carol_dalmeida@hotmail.com, raquelnogueiracordeiro@gmail.com.

3. Cirurgião geral. Professor assistente do departamento de cirurgia da Universidade Católica de Pernambuco - UNICAP e do Centro Universitário Maurício de Nassau - UNINASSAU. Mestrando em Ciências da Saúde na Universidade de Pernambuco - UPE. Email: dilaurentino@gmail.com.

Endereço para correspondência: Raquel Nogueira Cordeiro. Rua dos Coelhos, 300 - Boa Vista. Recife, PE, Brasil. CEP: 52.050-300. Email: raquelnogueiracordeiro@gmail.com. 


\section{INTRODUÇÃO}

A apendicite aguda é a principal causa de cirurgia abdominal de urgência na infância e adolescência, sendo incomum antes dos cinco anos ${ }^{1}$. O risco de desenvolver apendicite durante a vida é cerca de $8,6 \%$ para homens e $6,7 \%$ para mulheres ${ }^{2}$.

$\mathrm{O}$ apêndice vermiforme é um pequeno órgão tubular de fundo cego que se origina da parede póstero-medial do ceco, abaixo da válvula ileocecal, com comprimento que pode variar entre 2 e $20 \mathrm{~cm}^{3}$. É um órgão essencialmente móvel na maioria dos casos, fixado ao ceco através de apenas uma prega peritoneal, podendo assim se apresentar em diferentes posições ${ }^{4}$. As posições anatômicas mais comumente vistas na prática clínica são a retrocecal (atrás do ceco), a retrocólica (atrás da porção inferior do colo ascendente) e a pélvica (quando o apêndice fica pendurado por sobre a borda pélvica, em íntima relação com a tuba uterina e com o ovário direito em mulheres). Outras posições, a subcecal (abaixo do ceco), pré-ileal e pós-ileal (anterior ou posterior ao íleo terminal, respectivamente) e paracecal (ao lado do ceco), são ocasionalmente vistas ${ }^{5}$.

A situação do apêndice, por sua vez, é caracterizada pela sua relação com o ceco. Pode ser ascendente, descendente, externo ou interno. Quando o apêndice é ascendente, está na face posterior do ceco e do cólon, subindo para o rim e fígado. Quando é descendente, entra na pequena pelve. Quando é externo, se situa entre o ceco e entre a espinha anterossuperior e, quando interno, se situa em direção ao abdome para as alças do intestino delgado ${ }^{6}$. Essas variações anatômicas são de grande importância para o médico cirurgião, pois em função delas os sinais e sintomas podem variar ${ }^{7}$.

A sintomatologia clássica da apendicite está presente em $60 \%$ dos casos ${ }^{8}$. Em crianças e adolescentes, as manifestações clínicas alteram de acordo com a idade, variando de sintomas inespecíficos como irritabilidade, letargia e distensão abdominal em recém-nascidos até descrição detalhada dos sintomas e sinais clássicos da doença por adolescentes maiores ${ }^{9}$. A dor abdominal é o sintoma mais importante e o mais frequente da apendicite aguda, descrita inicialmente como cólica leve, com duração habitual de quatro a seis horas, localizada na região periumbilical ou epigástrica. À medida que a inflamação se propaga para a superfície do peritônio parietal, a dor localiza-se no quadrante inferior direito. A temperatura do corpo, na maioria das vezes, é normal ou ligeiramente aumentada $\left(37,2\right.$ à $\left.38^{\circ} \mathrm{C}\right)$. Em 50 a $60 \%$ das vezes surgem anorexia, náuseas e vômitos ${ }^{10}$.

A apresentação sintomatológica da apendicite depende, também, da posição anatômica do apêndice, podendo ocorrer dor abdominal atípica e ausência de hipersensibilidade abdominal. A alteração do hábito intestinal, desde diarreia até constipação intestinal, e queixas urinárias podem, por exemplo, ocorrer quando o apêndice situa-se adjacente à bexiga (posição pélvica) ${ }^{10}$. No entanto, poucos são os trabalhos que tem como objeto de estudo as variações anatômicas do apêndice e sua correlação com as diversas manifestações clínicas.

O desconhecimento sobre as diferentes formas que a doença venha a assumir em decorrência dessas variações ou a falta de experiência cirúrgica para lidar com essas variantes anatômicas, principalmente em crianças e adolescentes, pode levar a graves complicações intra ou pós-operatórias. Os sinais e sintomas clínicos inespecíficos na fase inicial da doença e as variações anatômicas do apêndice representam os principais entraves no reconhecimento clínico da apendicite, o que propicia atraso em relação ao diagnóstico e progressão da fase da doença ${ }^{6}$. Na fase inflamatória, o apêndice apresenta apenas sinais flogísticos, na fase necrótica há evidências de morte celular. Com a evolução da apendicite, a parede perde sua integridade e há perfuração com secreção purulenta, a qual pode estar apenas na topografia do apêndice (perfurada com peritonite localizada) ou estendida além da topografia (perfurada com peritonite generalizada) ${ }^{6}$. A letalidade nas apendicites não perfuradas é menor que $1 \%$, no entanto, pode ser superior a $5 \%$ em crianças com diagnóstico $\operatorname{tardio}^{11}$.

Nesse contexto, surgiu o interesse de realizar um estudo transversal em um centro de referência para emergência pediátrica com o objetivo de correlacionar as variedades da anatomia do apêndice com o diagnóstico de apendicite aguda e a fase da doença, bem como analisar as alterações na sintomatologia dos pacientes em decorrência dessas variações anatômicas.

\section{MÉTODO}

Trata-se de um estudo observacional, prospectivo, descritivo, tipo corte transversal. O estudo foi realizado no período de agosto de 2015 a julho de 2016 na Emergência Pediátrica do Hospital da Restauração, considerado centro de referência em emergência da cidade do Recife.

A amostra foi não probabilística, por conveniência, composta por 56 pacientes incluídos na faixa etária de 7 a 13 anos que foram admitidos no serviço de saúde mencionado e que tiveram o diagnóstico de apendicite aguda. Indivíduos com menos de 7 ou mais de 13 anos foram excluídos da pesquisa, assim como os que não tiveram diagnóstico confirmado. Os pesquisadores ficaram de plantão na emergência pediátrica durante 4 dias da semana. Os pacientes com suspeita de apendicite foram convidados a participar da pesquisa após explicação prévia dos objetivos. Posteriormente à assinatura do Termo de Consentimento Livre e Esclarecido, os participantes e responsáveis responderam às perguntas da primeira parte do formulário de pesquisa, as quais consistiam em informações sobre idade, sexo, raça, renda familiar, história de apendicite na família e manifestações clínicas. 
Além desses dados clínico-epidemiológicos, os achados do exame físico e da ultrassonografia (USG) também foram incluídos nessa primeira parte do formulário.

O exame físico foi realizado na presença do médico plantonista, com atenção especial à pesquisa dos sinais de Blumberg, de Rovsing e do Obturador. Durante essa etapa, os pacientes permaneciam em decúbito dorsal e eram questionados sobre a existência de dor que, caso positiva, deveria ter sua localização descrita.

Em alguns pacientes, a USG foi solicitada para auxiliar no diagnóstico e nesses casos, o pesquisador coletava os achados a partir do prontuário dos pacientes. Foi considerado achado positivo quando apresentava sinais diretos ou indiretos de apendicite aguda. Os sinais diretos consistiram na visualização do apêndice como uma imagem tubular imóvel hipoecoica, pouco compressível, com espessamento de parede, imagem em alvo com diâmetro maior que $6 \mathrm{~mm}$ e imagem hiperecogênica com sombra acústica compatível com apendicite. Já os sinais indiretos foram tidos como coleção líquida na luz do apêndice, líquido livre em fossa ilíaca direita e aumento da ecogenicidade da gordura mesentérica adjacente ${ }^{12}$.

Os indivíduos diagnosticados com apendicite foram encaminhados para o bloco cirúrgico e tiveram a apendicectomia realizada. Quando o diagnóstico não era confirmado, o paciente era retirado da pesquisa. Durante a operação, os pesquisadores obtiveram com os cirurgiões as informações sobre a posição do apêndice (retrocecal, retrocólica, paracecal, subcecal, pélvica, pré-ileal ou pós-ileal), sua situação em relação ao ceco (ascendente, descendente, externo ou interno) e a fase da apendicite (inflamatória, necrótica, perfurada com peritonite localizada ou perfurada com peritonite generalizada). Após a retirada do apêndice, os pesquisadores o mediram com uma régua. A partir dos dados coletados nessa etapa, foi preenchida a segunda parte do formulário de pesquisa.

$\begin{aligned} & \text { Os pesquisadores selecionaram os participantes na } \\ & \text { Emergência pediátrica do Hospital da Restauração, de acordo } \\ & \text { com os Critérios de Elegibilidade }(\mathrm{n}=56) \text {. }\end{aligned}$
$\begin{aligned} & \text { Preenchimento da primeira parte do formulário de pesquisa } \\ & \text { a partir de informações dos responsáveis, dos pacientes, de } \\ & \text { prontuários e de exame físico. }\end{aligned}$
$\square$
$\begin{aligned} & \text { Pacientes submetidos à apendicectomia e preenchimento da } \\ & \text { segunda parte do formulário. }\end{aligned}$

Fluxograma 1. Captação e seguimentos dos participantes da pesquisa

As informações obtidas durante o período da coleta foram armazenadas no banco de dados do programa Microsoft Excel 97-2003 com dupla entrada para verificar possíveis erros de digitação. Após verificação e correção de inconsistências, os dados foram analisados no Epi-info versão 7.1.5.2 e foram construídas tabelas de distribuição de frequências absolutas e relativas. O teste Qui-Quadrado foi utilizado para verificar as possíveis associações entre as variáveis, no qual foi considerado estatisticamente significante quando $\leq 0,05$.

Esse projeto atendeu aos requisitos da Comissão Nacional de Ética e Pesquisa (CONEP) do Conselho Nacional de Saúde do Ministério da Saúde do Brasil (CNS/ MS). A coleta de dados foi iniciada mediante a aprovação do Comitê de Ética em Pesquisa em Seres Humanos da FPS, com Certificado de Apresentação para Apreciação Ética (CAAE) $\mathrm{n}^{\circ} 47942615.0 .0000 .5569$ e protocolo $\mathrm{n}^{\circ}$ $075822 / 2015$.

\section{RESULTADOS}

Um total de 56 pacientes, incluídos na pesquisa com o diagnóstico de apendicite aguda, tiveram seus dados clínico-epidemiológicos e resultados de exame de imagem coletados. A amostra foi composta em sua maioria por indivíduos do sexo masculino $(62,5 \%)$ e restringiu-se à faixa etária de 7 a 13 anos, com moda de 9 anos (23,2 $\%)$ e média de 10,19 anos $(\sigma=2,08)$. Em relação à raça autodeclarada, o predomínio foi de pardos $(60,7 \%)$ e brancos $(37,5 \%)$, não houve nenhum integrante indígena ou amarelo. A maior parte da amostra $(71,4 \%)$ apresentava renda familiar de 1 a 3 salários mínimos. A ultrassonografia (USG) foi solicitada em 44 pacientes, com achados positivos para apendicite em $84,1 \%$. As características gerais da amostra estão disponíveis na Tabela 1 .

Tabela 1 - Características clínico-epidemiológicas e exame de imagem dos pacientes pediátricos com apendicite aguda

\begin{tabular}{lcc}
\hline Variáveis & N=56 & $\mathbf{\%}$ \\
\hline Sexo & & \\
Masculino & 35 & 62,5 \\
Feminino & 21 & 37,5 \\
Idade* & & \\
$<10$ anos & 25 & 44,6 \\
$\geq 10$ anos & 31 & 55,4 \\
Raça & & \\
Parda & 34 & 60,7 \\
Branca & 21 & 37,5 \\
Negra & 1 & 1,8 \\
& & \\
Indígena/Amarela & 0 & \\
Renda Familiar & & \\
$<1$ Salário Mínimo & 16 & 28,6 \\
$\geq 1$ Salário Mínimo & 40 & 71,4 \\
História Familiar (Apendicite) & & \\
Sim & 23 & 41,1 \\
Não & 33 & 58,9 \\
USG & & \\
Positiva & 37 & 84,1 \\
Negativa & 7 & 15,9 \\
*A idade foi estratificada em < 10 anos (infância) e $\geq 10$ anos \\
(adolescência) de acordo com a Organização Mundial da Saúde (OMS) \\
que preconiza adolescência em indivíduos de 10 a 19 anos
\end{tabular}


Aguiar AA, et al. Avaliação das posições e situações anatômicas do apêndice em pacientes pediátricos.

Os dados da Tabela 2 mostram as características do apêndice vermiforme coletadas durante a apendicectomia. A situação do apêndice mais encontrada foi a descendente $(46,43 \%)$ e a posição foi a pélvica $(37,5 \%)$. Verificou-se que a maioria dos pacientes teve apendicite diagnosticada e operada ainda na fase inflamatória da doença $(48,2 \%)$. O comprimento do apêndice teve média de $7,7 \mathrm{~cm}$, com média de 7,8 cm nos homens e 7,4 cm nas mulheres.

Os dados da Tabela 3 mostram a relação da posição do apêndice com o quadro clínico, evidenciando em que posição cada sintoma é mais frequente. As manifestações clínicas mais encontradas foram dor em fossa ilíaca direita, positiva em todos os pacientes, vômitos, náuseas e febre. Sintomas menos comuns como constipação, diarreia e disúria foram identificados em mais ou menos $1 / 4$ da população.

Em relação aos sinais sugestivos de peritonite, o sinal de Blumberg foi testado em todos os pacientes e a posição pélvica foi a que apresentou maior percentual (38\%) dentre os achados positivos (89.3\%). Já o sinal de Rovsing, do total dos 56 pacientes, não foi testado em 12 e o sinal do Obturador, em 16. Dos achados positivos, o sinal de Rovsing (verificado em 29.5\% dos testados) foi mais encontrado na posição pélvica e o sinal do Obturador (positivo em 30\% dos testados) nas posições pélvica e retrocecal, ambas apresentando a mesma frequência. A incidência dos sinais de acordo com cada posição está detalhada na Tabela 4.
Tabela 2 - Características do apêndice vermiforme em pacientes pediátricos com apendicite aguda

\begin{tabular}{l|c|c}
\hline Variáveis & $\mathbf{N = 5 6}$ & $\mathbf{\%}$ \\
\hline Fase da apendicite & & \\
\hline Não complicada & 33 & 58,9 \\
\hline Inflamatório & 27 & 48,2 \\
\hline Necrótica & 6 & 10,7 \\
\hline Complicada & 23 & 41,1 \\
\hline Perfurado com peritonite localizada & 14 & 25 \\
\hline Perfurado com peritonite & 9 & 16,1 \\
\hline generalizada & & \\
\hline Situação do apêndice & 16 & 28,6 \\
\hline Ascendente & 20 & 46,4 \\
\hline Descendente & 11 & 19,6 \\
\hline Interna & 3 & 5,4 \\
\hline Externa & & \\
\hline Posição do apêndice & 16 & 28,6 \\
\hline Retrocecal & 0 & 0 \\
\hline Retrocólica & 21 & 37,5 \\
\hline Pélvica & 5 & 8,9 \\
\hline Subcecal & 6 & 10,7 \\
\hline Pré-ileal & 5 & 8,9 \\
\hline Pós-ileal & 3 & 5,4 \\
\hline Paracecal & &
\end{tabular}

Tabela 3 - Manifestações clínicas dos pacientes pediátricos com apendicite aguda

\begin{tabular}{lcccccccc}
\hline & \multicolumn{7}{c}{ Sinais e Sintomas } \\
\cline { 2 - 9 } & $\begin{array}{c}\text { Dor em } \\
\text { FID }\end{array}$ & Vômitos & Náuseas & Febre & Anorexia & Disúria & Diarreia & Constipação \\
\cline { 2 - 9 } Posições & $\%$ & $\%$ & $\%$ & $\%$ & $\%$ & $\%$ & $\%$ & $\%$ \\
\hline Retrocecal & 28,6 & 34,1 & 29 & 23,3 & 11,1 & 18,7 & 35,7 & 0 \\
Pélvica & 37,5 & 34,1 & 41,9 & 43,3 & 48,1 & 50 & 50 & 58,3 \\
Subcecal & 8,9 & 9,7 & 3,2 & 10 & 7,4 & 12,5 & 0 & 16,7 \\
Pré-ileal & 10,7 & 9,7 & 16,1 & 10 & 18,5 & 6,3 & 7,1 & 16,7 \\
Pós-ileal & 8,9 & 7,3 & 3,2 & 6,7 & 11,1 & 12,5 & 7,1 & 0 \\
Paracecal & 5,3 & 4,8 & 6,4 & 6,7 & 3,7 & 0 & 0 & 8,3 \\
Total (N) & 56 & 41 & 31 & 30 & 27 & 16 & 14 & 12 \\
\hline
\end{tabular}

*A posição retrocólica não foi encontrada nos indivíduos desse estudo.

Tabela 4 - Sinais sugestivos de peritonite em pacientes pediátricos com apendicite aguda

\begin{tabular}{lccc}
\hline & \multicolumn{3}{c}{ Sinais } \\
\cline { 2 - 4 } Posições & Blumberg & Rovsing & Obturador \\
\hline Retrocecal & $\mathrm{N}(\%)$ & $\mathrm{N}(\%)$ & $\mathrm{N}(\%)$ \\
Pélvica & $13(26)$ & $3(23,1)$ & $4(33,3)$ \\
Subcecal & $19(38)$ & $6(46,1)$ & $4(33,3)$ \\
Pré-ileal & $5(10)$ & $1(7,7)$ & $2(16,7)$ \\
Pós-ileal & $5(10)$ & $2(15,4)$ & $1(8,3)$ \\
Paracecal & $5(10)$ & 0 & $1(8,3)$ \\
\hline Total & $3(6)$ & $1(7,7)$ & 0 \\
\hline
\end{tabular}

*A posição retrocólica não foi encontrada nos indivíduos desse estudo. 
Em todas as posições, a fase da apendicite mais frequente foi a inflamatória, exceto na posição subcecal, em que $60 \%$ dos apêndices foram encontrados na fase perfurada. As demais frequências das fases da apendicite relacionadas com cada posição podem ser vistas na Tabela 5 .

A possível associação entre a posição do apêndice e a fase da apendicite e entre a posição do apêndice e sinais clínicos foi verificada através da aplicação do teste Quiquadrado. Entre a posição subcecal e a fase da apendicite complicada não houve associação estatisticamente significante $(p=0,367)$. Também não se observou significância na relação da posição pélvica com o sinal do Obturador $(\mathrm{p}=0,398)$, nem com disúria $(\mathrm{p}=0,221)$ ou constipação $(p=0,092)$.

Tabela 5 - Fase da Apendicite de acordo com cada posição

\begin{tabular}{lccccc}
\hline & \multicolumn{5}{c}{ Fase da Apendicite } \\
\cline { 2 - 5 } Posição & Inflamatória & Necrótica & $\begin{array}{c}\text { Perfurada com } \\
\text { peritonite localizada }\end{array}$ & $\begin{array}{c}\text { Perfurada com } \\
\text { peritonite generalizada }\end{array}$ & Total \\
\hline Retrocecal & $\mathrm{N}(\%)$ & $\mathrm{N}(\%)$ & $\mathrm{N}(\%)$ & $\mathrm{N}(\%)$ & $16(100)$ \\
Retrocólica & $7(43,7)$ & 0 & $3(18,7)$ & 0 & 0 \\
Pélvica & $9(42,9)$ & $2(9,5)$ & 0 & $4(19)$ & $21(100)$ \\
Subcecal & $2(40)$ & 0 & $3(28,6)$ & 0 & $5(100)$ \\
Pre-ileal & $4(66,6)$ & 0 & $1(16,7)$ & $1(16,7)$ & $6(100)$ \\
Pos- ileal & $3(60)$ & 0 & $1(20)$ & 0 & $5(100)$ \\
Paracecal & $2(66,7)$ & $1(33,3)$ & 0 & $9(16,1)$ & $3(100)$ \\
Total & $27(48,2)$ & $6(10,7)$ & $14(25)$ & $56(100)$ \\
\hline
\end{tabular}

\section{DISCUSSÃO}

No presente estudo, a apendicite aguda foi diagnosticada com maior incidência no sexo masculino $(62,5 \%)$, em concordância com o estudo de Borges et al. ${ }^{16}$, o qual envolveu crianças e adolescentes admitidos na emergência pediátrica do IMIP com apendicite aguda no ano de 2002, onde cerca de 53\% eram do sexo masculino. Outros estudos também identificaram o predomínio de meninos nos pacientes com apendicite, como o de Hall et al. ${ }^{15}$, realizado nos EUA em 2007 e o de Vital e Martins ${ }^{16}$ em São Paulo no ano de 2005. Esse achado ratifica a continuidade do perfil epidemiológico quanto a prevalência da apendicite aguda no sexo masculino.

Em relação à história familiar de apendicite, no nosso estudo, a maioria dos pacientes $(58,9 \%)$ teve esta variável negativa, o que também foi visto no estudo já referido de Borges et al. ${ }^{14}$, onde a maioria $(65,8 \%)$ dos pacientes não relatou história familiar de apendicite. No entanto, conforme o estudo de Gauderer et al. ${ }^{17}$, envolvendo crianças e adolescentes na faixa etária de 2 a 19 anos submetidas a apendicectomia, em CS, EUA, história de apendicite entre familiares de primeiro grau é um possível fator de risco. No nosso trabalho, por não possuirmos grupo controle de pacientes sem apendicite, não pudemos verificar o risco relativo, ficando para investigação em futuros trabalhos.

Quanto aos exames complementares de imagem, a ultrassonografia foi a mais solicitada. A USG se mostra um bom método de apoio diagnóstico em crianças pelo seu baixo custo, técnica sem radiação ionizante e relativamente simples, bem tolerada e pode ser usada como método de primeira escolha ${ }^{12}$. Dos pacientes submetidos ao exame, $84,1 \%$ apresentaram resultado positivo, sendo similar à pesquisa de Vital e Martins ${ }^{16}$, na qual a USG ajudou de maneira favorável o diagnóstico de $80.1 \%$ dos casos em que o exame foi indicado, o que fortemente ratifica a utilidade de tal exame no diagnóstico da apendicite.

No presente estudo, no qual foram avaliados pacientes pediátricos submetidos à laparotomia de emergência por apendicite aguda, a posição pélvica foi a mais frequente, o que é condizente com os resultados dos trabalhos, também realizados em pacientes com apendicite, de Ahmed et al. ${ }^{3}$ (envolvendo pacientes na faixa etária de 18 a 93 anos) e Denjalic et al. ${ }^{19}$ (sem indicação da faixa etária na sua metolodologia).

No entanto, outros estudos sobre frequência das posições do apêndice em autópsia de indivíduos adultos sem infecção, encontraram a posição retrocecal como a mais evidenciada ${ }^{19,20,21}$. Uma das explicações viáveis para essa diferença entre posição do apêndice com a existência ou não de infecção seria uma possível menor probabilidade de inflamação do apêndice na posição retrocecal ${ }^{22}$, o que pode ter contribuído para o maior achado dessa posição na autópsia de indivíduos sem apendicite. Porém, isto está em contradição com outros estudos realizados também em cadáveres de pessoas que não tiveram apendicite, onde a posição pélvica foi a mais frequente ${ }^{24,26}$. Assim, pesquisas futuras poderão elucidar esse aparente conflito de resultados.

Em relação às manifestações clínicas, as mais encontradas nesse estudo foram dor em fossa ilíaca direita, 
Aguiar AA, et al. Avaliação das posições e situações anatômicas do apêndice em pacientes pediátricos.

vômitos, náuseas e febre, independentemente da posição. Dentre os sintomas menos frequentes tivemos constipação, diarreia e disúria. Em 50\% dos pacientes com disúria, o apêndice correspondia à posição pélvica, concordando com o que é referido na literatura ${ }^{25}$, onde é apontado que a presença de dor ao urinar é mais relatada naquela posição. Além disso, $58 \%$ dos pacientes com constipação apresentaram o apêndice também na posição pélvica, não tendo sido encontrado estudos correlacionando esse sintoma com essa posição. Apesar dos sintomas variarem em sua frequência nas posições, não se observou associação entre a posição pélvica e disúria e entre aquela e constipação. Uma hipótese para não evidenciarmos associação significativa seria atribuída a pequena amostra do presente trabalho.

Quanto aos sinais sugestivos de peritonite, verificouse que o de Blumberg foi positivo em $89.3 \%$ dos casos, o que aponta no sentido de ser este teste útil para o diagnóstico da apendicite. Já o sinal de Rovsing resultou positivo em $29,5 \%$ dos casos em que foi testado (44), sugerindo não ser esse um bom teste para diagnóstico. $\mathrm{O}$ sinal do Obturador, por sua vez, foi encontrado em maior frequência na posição pélvica e retrocecal. Jerry et al. ${ }^{23}$ cita uma relação entre o sinal do Obturador e a posição pélvica devido à própria anatomia, pois este sinal é realizado com a rotação interna do membro inferior direito, apresentando assim uma maior proximidade com o apêndice pélvico. Entretanto, não há estudos com metodologias definidas que comprovem associação da posição pélvica com o sinal do Obturador. Da mesma forma, em nosso estudo, o teste do Qui-quadro aplicado para estas variáveis não evidenciou associação estatisticamente significante $(\mathrm{p}=0,398)$.

No nosso estudo, o comprimento do apêndice foi maior nos homens do que nas mulheres, o que condiz com os resultados dos trabalhos de Gholalipour et al. ${ }^{21}$ e Ghorbani et al. ${ }^{26}$ mas difere dos resultados dos estudos de Rahman et al. ${ }^{25}$ e Bakheit e Warille ${ }^{27}$ que encontraram um tamanho maior do apêndice nas mulheres do que nos homens.

No que se refere à fase da apendicite, constatouse que, em todas as posições, a inflamatória foi a mais frequente, exceto na subcecal, onde $60 \%$ dos apêndices estavam na fase perfurada. No entanto, não houve associação estatisticamente significante entre a posição subcecal e a fase da apendicite complicada $(p=0,367)$. Atribuímos esse resultado a um viés decorrente da pequena amostra utilizada neste estudo. Assim, entendemos pertinente que, em uma futura pesquisa, seja investigado se existe uma correlação entre a fase perfurada e a posição subcecal.

Nosso estudo tem as seguintes limitações: trata-se de uma casuística muito pequena, o que limita tomar as conclusões como definitivas. Em conclusão, este estudo tem a possibilidade de contribuir para o conhecimento epidemiológico da apendicite em crianças, bem como, facilitar o diagnóstico baseado nos sinais e sintomas da doença, trazendo maior efetividade para as condutas médicas adotadas. Assim, sugere-se a realização de novos estudos nessa área, sobretudo, devido à relevância do tema e escassez de pesquisas com esse tipo de delineamento.

\section{Apêndice: Formulário de Pesquisa}

DATA: .........................

$\mathrm{N}^{\circ}$ do formulário: ............ Pesquisador:

A) IDENTIFICAÇÃO

Nome:

$\mathrm{N}^{\mathrm{o}}$ do prontuário: .............................

Data de Nascimento: ............................

B) CARACTERÍSTICAS (a partir de informações coletadas)

1. Idade:

2. Sexo: ( ) M ( ) F

3. Raça: ( ) Branca () Parda ( ) Amarela ( ) Negra

4. Renda Familiar : () menos de 1 salário mínimo () de 1 a 3 salários mínimos ( ) de 3 a 6 salários mínimos ( ) de 6 a 10 salários mínimos ( ) mais de 10 salários mínimos

\section{História familiar de apendicite: ( ) Sim ( ) Não}

Se sim: Quem? (Pode selecionar mais de uma opção)

( ) mãe ( ) pai ( ) irmão ( ) irmã ( ) avô ( ) avó

( ) Outros: ..............

\section{Sinais e sintomas:}

() febre () anorexia () dor na fossa ilíaca direita () náuseas () vômitos ( ) disúria ( ) constipação intestinal ( ) diarreia ( ) sinal de Blumberg () sinal de Rovsing ( ) sinal do obturador

7. USG

() Solicitado

( ) Positivo

() Negativo

( ) Não solicitado

8. Situação do apêndice: ( ) ascendente ( ) descendente ( ) interna ( ) externa

9. Posição do apêndice: ( ) retrocecal ( )retrocólica ( ) pélvica ( ) subcecal ( ) pré-ileal

() pós-ileal ( ) paracecal

10. Comprimento do apêndice: $\mathrm{cm}$

11. Fase da apendicite:

- Inflamatória ( )

- Necrótica $($ )

- Perfurada com peritonite localizada ( )

- Perfurada com peritonite generalizada ( )

Participação dos autores: Informamos para devido fins que o artigo foi confeccionado em conjunto pelo grupo de autores com o grau de participação seguinte: Coleta de dados (MGDM Gusmão, ALL Mello, BAA Velloso, CVM D'Almeida - Acadêmicas de Medicina). Orientação organizacional e sobre a essência, argumentação e relevância do trabalho: (AA Aguiar-Médico cirurgião-pediátrico). Análise, pesquisa dos artigos, leitura e exclusão de pesquisas não pertinentes ao envolvimento do tema escolhido: (AA Aguiar). Leitura e escrita do conteúdo: (AA Aguiar, MGDM Gusmão, ALL Mello, BAA Velloso, CVM D’Almeida, RN Cordeiro, DL Lima). Revisão do texto quanto a integridade e veracidade quanto as fontes utilizadas: (AA Aguiar, DLLima, RN Cordeiro). Dessa forma, o grupo de autores certifica participação conjunta na confecção do artigo, esperando contribuir no tema em questão. 


\section{REFERÊNCIAS}

1. Impellezzeri P, Centoze A, Antonuccio P, Turiaco N, Basile $\mathrm{M}$, Argento S, Argento S, Romeo C. Utility of a scoring system in the diagnosis of acute appendicitis in pediatric age. A retrospective study. Minerva Chir. 2002;57(3):341-6.

2. Adiss DG, Shaffer N, Fowler BS, et al. The epidemiology of appendicitis and appendectomy in the United States. Am J Epidemiol. 1990;132:910-25.

3. Ahmed I, Asgeirsson KS, Beckingham IJ, Lobo DN. The position of the vermiform appendix at laparoscopy. Surg Radiol Anat. 2007;29:165-8. doi: 10.1007/s00276-007-0182-8.

4. Wakeley CPG. The position of the vermiform appendix as ascer-tained by an analysis of 10,000 cases. J Anat. 1933;67:277-83. Available from: https://www.ncbi.nlm.nih. gov/pmc/articles/PMC1249345/.

5. Standring S. Anatomia: a base anatômica da prática clínica. 40a ed. São Paulo: Elsevier; 2010.

6. Jacob $\mathrm{O}$, Testut L. Tratado de anatomía topográfica con aplicaciones medicoquirúrgicas. 8a ed. Barcelona: Salvat; 1977.

7. Okano N, Vargas EC. Apendicite aguda. Medicina (Ribeirão Preto). 1995;18(4):676-80.

8. Ma KW, Chia NH, Yeung HW, Cheung MT. If not appendicitis, then what else can it be? A retrospective review of 1492 appendecto-mies. Hong Kong Med J. 2010;16:127. Available from: https://www.hkmj.org/system/files/ hkm1002p12.pdf.

9. Rothroc SG, Pagane J. Acute appendicitis in children: emergency departament diagnosis and management. Ann Emerg Med. 2001;36:39-51. doi: 10.1067/mem.2000.105658.

10. Guidry SP, Poole VG. The anatomy of appendicitis. Am Surg. 1994;60:68-71.

11. Hardin DM Jr. Acute appendicitis: review and update. Am Fam Phys. 1999;60:2027-34. Available from: https://www. aafp.org/afp/1999/1101/p2027.html.

12. Torres OJM, Lins AAL, Nunes PMS, Corrêa FCF, Carvalho Júnior OS, Castro FC. Avaliação ultra-sonográfica da apendicite aguda. Rev Col Bras Cir. 2001;28(1):39-43. http:// dx.doi.org/10.1590/S0100-69912001000100008.

13. Eisenstein E. Adolescência: definições, conceitos e critérios. Adolesc Saude. 2005;2(2):6-7. Disponível em: http://www. adolescenciaesaude.com/detalhe_artigo.asp?id=167.

14. Borges PSGN, Lima MC, Falbo Neto GH. Validação do escore de Alvarado no diagnóstico de apendicite aguda em crianças e adolescentes no Instituto Materno Infantil de Pernambuco, IMIP. Rev Bras Saude Mater Infant. 2003;3(4):439-45. http:// dx.doi.org/10.1590/S1519-38292003000400008.

15. Hall MJ, DeFrances CJ, Williams MPH, Golosinskiy A, Schwartzman A. National Hospital Discharge Survey:
2007 Summary. Natl Health Stat Report. 2010;(29):1-20, 24. Available from: https://www.cdc.gov/nchs/data/nhsr/ nhsr029.pdf.

16. Vital Jr PF, Martins JL. Estado atual do diagnóstico e tratamento da apendicite aguda na criança: avaliação de 300 casos. Rev Col Bras Cir. 2005;32(6):310-5. http://dx.doi. org/10.1590/S0100-69912005000600005.

17. Gauderer MW, Crane MM, Green JA, DeCou JM, Abrams RS. Acute appendicitis in children: the importance of family history. J Pediatr Surg. 2001;36(8):1214-7. doi: 10.1053/ jpsu.2001.25765.

18. Denjali'c A, Deli'c J, Deli'c-Custendil S, Muminagi'c S. Variations in position and place of formation of appendix vermiformis found in the course of open appendectomy. Med Arh. 2009;63(2):100-1.

19. Souza SC, Costa SRMR, Souza IGS. Vermiform appendix: positions and length - a study of 377 cases and literature review. J Coloproctol (Rio J.). 2015;35(4):212-6. http:// dx.doi.org/10.1016/j.jcol.2015.08.003.

20. Paul UK, Naushaba H, Begum, Alam J. Position of vermiform appendix: a postmortem study. Bangladesh J Anat. 2009;7(1):34-6. https://doi.org/10.3329/bja.v7i1.3015.

21. Golalipour MJ, Arya B, Azarhoosh R, Jahanshahi M. Anatomical variations of vermiform appendix in SouthEast Caspian Sea (Gorgan-Iran). J Anat Soc India. 2003;52(2):141-3. Available from: http://medind.nic.in/jae/ t03/i2/jaet03i2p141.pdf.

22. Clegg-Lamptey JN, Armah H, Naaeder SB, Adu-Aryee NA. Position and susceptibility to inflammation of vermiform appendix in Accra, Ghana. East Afr Med J. 2006;83(12):670-3.

23. Old JL, Dusing RW, Yap W, Dirks J. Imaging for suspected appendicitis. Am Fam Phys. 2005;71(1):71-8. Available from: https://www.aafp.org/afp/2005/0101/p71.html.

24. Rahman MM, Khalil M, Rahman H, Mannan S, Sultana SZ, Ahmed S. Anatomical positions of vermiform appendix in Bangladeshi people. J Bangladesh Soc Physiol. 2006;1:5-9. https://doi.org/10.3329/jbsp.v1i0.713.

25. Ojeifo JO, Ejiwunmi AB, Iklaki J. The position of the vermiform appendix in Nigerians with a review of the literature. West Afr J Med. 1989;8(3):198-204.

26. Ghorbani A, Forouzesh M, Mohammad Kazemifar A. Variation in anatomical position of vermiform appendix among Iranian population: an old issue which has not lost its importance. Anat Res Int. 2014:Article ID 313575. Doi:10.1155/2014/313575.

27. Bakheit MA, Warille AA. Anomalies of the vermiform appendix and prevalence of acute appendicitis in Khartoum. East Afr Med J. 1999;76(6):338-40.

Recebido: 24.07 .18

Aceito: 05.02 .19 